\title{
INVESTIGATION ON DISSOLUTION PATTERN AND MATHEMATICAL MODELING OF DRUG RELEASE OF QUERCETIN BY COMPLEXATION WITH CYCLODEXTRIN NANOSPONGES
}

\author{
SOBITHARANI ${ }^{1 *}$, ANANDAM S ${ }^{1}$, MOHAN VARMA M ${ }^{2}$, VIJAYA RATNA J ${ }^{3}$, SHAILAJA P ${ }^{3}$ \\ ${ }^{1}$ Department of Vijaya College of Pharmacy, Hyderabad, Telangana, India. ${ }^{2}$ Department of Pharmaceutical Technology, Shri Vishnu \\ College of Pharmacy, Bhimavaram, Andhra Pradesh, India. ${ }^{3}$ Department of Andhra University College of Pharmaceutical Sciences, \\ Visakhapatnam, Andhra Pradesh, India. Email: sobhitarani@gmail.com
}

Received: 28 February 2019, Revised and Accepted: 10 April 2019

ABSTRACT

Objective: The main objective of this study was to investigate the release pattern of a poorly water-soluble drug quercetin (QU) by fabricating its cyclodextrin nanosponges.

Methods: Characterization of the original QU powder and QU-loaded nanosponges was carried out by the Fourier-transformed infrared (FTIR) spectroscopy, transmission electron microscopy (TEM), and dissolution tester. The drug release pattern was subjected to various kinetic models.

Results: FTIR studies confirmed the formation of inclusion complex of drug. The particle size analysis revealed that the average particle size measured by laser light scattering method is around 400-420 nm with low polydispersity index. The particle size distribution is unimodal and having a narrow range. A sufficiently high zeta potential indicates that the complexes would be stable and the tendency to agglomerate would be miniscule. TEM image revealed the porous nature of nanosponges. The dissolution of the QU nanosponges was significantly higher compared with the pure drug.

Conclusion: From the kinetic study, it is apparent that the regression coefficient value closer to unity in case of Korsmeyer-Peppas model indicates that the drug release exponentially to the elapsed time. n value obtained from the Korsmeyer-Peppas plots, i.e., 0.9911 indicating non-Fickian (anomalous) transport; thus, it projected that delivered its active ingredient by coupled diffusion and erosion.

Keywords: Nanosponges, Cyclodextrins, Complexation, Dissolution, Release kinetics, Quercetin.

(C) 2019 The Authors. Published by Innovare Academic Sciences Pvt Ltd. This is an open access article under the CC BY license (http://creativecommons. org/licenses/by/4. 0/) DOI: http://dx.doi.org/10.22159/ajpcr.2019.v12i5.32864

\section{INTRODUCTION}

Flavonoids are a group of phenolic compounds that are widely distributed in different plant foods such as fruits, vegetables, leaves, and grains. There are different classes of flavonoids, for example, flavones, flavonones, isoflavones, flavonols, flavanonols, and anthocyanins [1,2]. Quercetin (QU) $\left(3,3^{\prime}, 4^{\prime}, 5,7\right.$-pentahydroxyflavone) is an abundantly distributed plant; flavonol shows a wide range of pharmacological activities such as antioxidant, radical scavenging, and anticarcinogenic activity and is helpful in the recovery of n-diethyl nitrosamine-induced carcinogenesis $[3,4]$, human leukemia cell [5], streptozotocin-induced diabetes [6], chronic renal failure, and reactive oxygen species-induced DNA damage [7]. QU and other flavonoids have been shown to modify eicosanoid biosynthesis, protect low-density lipoprotein from oxidation, prevent platelet aggregation, and promote relaxation of cardiovascular smooth muscle in antihypertensive and antiarrhythmic effects. In spite of this wide spectrum of pharmacological properties, its use in pharmaceutical field is limited by its low water solubility. QU is extremely hydrophobic in nature and has such poor absorption in the gastrointestinal tract. QU has a low solubility $(7.7 \mu \mathrm{g} / \mathrm{mL}$ in water, $5.5 \mu \mathrm{g} / \mathrm{mL}$ in simulated gastric fluid, and $28.9 \mu \mathrm{g} / \mathrm{mL}$ in simulated intestinal fluid), which contributes to a low absorption in vivo. Its oral bioavailability is $<17 \%$ in rats, even $1 \%$ in men [8-12]. As a result, the clinical application of the drug is greatly restricted. All these highlight the need for an improved formulation for $\mathrm{QU}$ with enhanced dissolution so that its absorption can be greatly enhanced. To overcome the above problems of QU and to achieve better clinical efficacy, many new drug delivery systems have been studied and developed in recent years, of which nanodrug delivery system has become a research hotspot with their numerous advantages such as reducing particle size, enhancing surface area, increasing drug permeability, improving intracorporeal circulation and distribution of the drug, and enhancing drug targeting and so on [13].

Cyclodextrins (CDs) are cyclic oligosaccharides joined to each other by $\alpha$ - $(1 \rightarrow 4)$-linkages and synthesized by the enzymatic degradation of hydrolyzed starch. They consist of both hydrophilic inclusion complexes with functional groups on lipophilic compounds in aqueous solution [14]. The use of CDs represents another novel technological approach to increase drug solubility, stability, and oral bioavailability $[15,16]$. However, the inclusion constants of native CDs rarely exceed the value of $10^{3}$. The cavity size of $\alpha-C D$ is insufficient for many drugs and $\gamma$-CD is expensive $[17,18]$. In general, $\beta$-CD has weaker complexing ability than conventional CDs. Among all CDs, $\beta$-CD is receiving attention due to its low cost and cavity size suitable for the widest range of drugs in aqueous media [19]. However, the low aqueous solubility and nephrotoxicity limited the use of $\beta-C D$, especially in parenteral drug delivery. Recently, CD-based nanosponges have emerged as a promising drug delivery platform for recalcitrant, difficult-to-formulate molecules [20-22].

Nanosponges are recently developed hypercross-linked CD polymers nanostructured to form three-dimensional networks; they are obtained by reacting $\mathrm{CD}$ with a suitable cross-linking agents. CD-based nanosponges showed superior complexing ability than natural CDs toward many molecules. They have been used to increase the solubility of poorly soluble actives, to protect the labile groups, and to control the release [23]. The present study was designed to investigate the feasibility of utilizing the CD-based nanosponges to improve the aqueous solubility and dissolution characteristics of QU. 


\section{MATERIALS AND METHODS}

Materials

QU was purchased from Sigma-Aldrich, Singapore. CD nanosponges were prepared in our laboratory as reported elsewhere [24]. All other reagents used in the study were of reagent grade.

\section{Methods}

Preparation of QU-loaded nanosponges (QUNS)

Drug-loaded nanosponges were prepared by lyophilization technique as previously reported. Accurately weighed quantities of nanosponges were suspended in $50 \mathrm{~mL}$ of Milli-Q water using a magnetic stirrer, and then, the excess amount of QU was added and the mixture was sonicated for $10 \mathrm{~min}$ and was kept for $24 \mathrm{~h}$ under stirring. The suspensions were centrifuged at $2000 \mathrm{rpm}$ for $10 \mathrm{~min}$ to separate the uncomplexed drug as a residue below the colloidal supernatant. The supernatant was lyophilized on a lyophilizer (LARK INDIA) at $-20^{\circ} \mathrm{C}$ temperature and operating pressure 13.33 mbar. The dried powder was stored in a desiccator [25].

\section{Determination of QU loading in NS}

Weighed amount of QUNS complexes was dissolved in methanol, sonicated for 10 min to break the complex, diluted suitably, and then analyzed by ultraviolet (UV) spectrophotometer at $372 \mathrm{~nm}$.

We used a previously reported formula to calculate "percent drug association:"

$$
\frac{C^{\prime} M^{\prime} 100}{T^{\prime} W}
$$

Where, $C$ is the concentration of QU measured in $\mathrm{mg}, M$ is the total particle mass - the yield of the formulation, $T$ is the particle mass tested - weight of NS formulation used for quantification of QU, and $W$ is the initial weight of the drug fed for loading - known amount of drug used for formulation.

\section{Characterization of QU nanosponge complexes (QUNS)}

The Fourier transformed infrared (FTIR) spectra of $\beta$-CD, plain nanosponges (NS2), QU, and QUNS complexes (QUNS2) were carried out by potassium bromide disk method using Tensor 27 FTIR spectrophotometer (Bruker Optics, Germany) in the region of 4000-600 $\mathrm{cm}^{-1}$. The particle size distribution was observed using a Mastersizer 2000 (Malvern Instruments Ltd., Worcestershire, UK) and the zeta potential of the same was determined using a Zetasizer (Malvern Instruments Ltd., Worcestershire, UK). The morphology of the QUNS was observed under transmission electron microscopy (TEM) (JEM-2000 EXII; JEOL, Tokyo, Japan).

\section{Dissolution studies}

The in vitro dissolution study of QU was carried out using multicompartment $(\mathrm{n}=6)$ rotating cells with a dialysis membrane (Sartorius cutoff 12,000 Da). The donor phase consisted of formulations containing $5 \mathrm{mg}$ of QU in $100 \mathrm{ml}$ simulated gastric fluid ( $\mathrm{pH}$ 6.4). The receptor phase also contains the same medium. The receptor phase was added with $0.5 \% \mathrm{w} / \mathrm{v}$ sodium lauryl sulfate $(1 \mathrm{ml})$ to maintain proper sink conditions. The receptor phase was withdrawn completely after fixed time intervals, suitably diluted with distilled water and was analyzed using UV spectrophotometer at $372 \mathrm{~nm}$. The experiment was carried out in triplicate.

\section{Drug release kinetics}

To elucidate the mode and mechanism of drug release, the data from the in vitro dissolution study were fitted into various mathematical models such as zero order, first order, Higuchi's, and Korsmeyer-Peppas model. The release data from the nanosponge formulation were determined by curve fitting method. Data obtained from in vitro release studies were fitted to various kinetic equations.

Zero-order model:

$$
Q_{t}=Q_{0}+K_{0} t
$$

Where, $Q_{t}$ is the amount of drug dissolved in time $t, Q_{0}$ is the initial amount of drug in the solution, and $K_{0}$ is the zero-order release constant.

First-order model:

$$
\ln \left(Q_{\infty}-Q_{t}\right)=\ln Q_{0}+K_{t}
$$

Where, $Q_{t}$ is the amount of drug dissolved in time $t, Q_{0}$ is the initial amount of drug in the solution, $Q_{\infty}$ is the amount release in time $\infty$ (100\% drug release), and $K$ is the first-order release constant.

Higuchi model:

$$
Q_{t}=k_{H} t^{1 / 2}
$$

Where, $Q_{t}$ is the amount of drug dissolved in time $t$ and $k_{H}$ is the Higuchi dissolution constant.

Korsmeyer-Peppas model (power law):

$$
\frac{Q_{t}}{Q_{¥}}=K_{K} t^{n}
$$

Or

$$
\log Q_{t}=\log K_{k}+n \log t
$$

Where, $Q_{t}$ is the amount of drug dissolved in time $t, Q_{\infty}$ is the amount release in time $\infty, K_{k}$ is the rate constant, and $\mathrm{n}$ is the diffusional exponent, this indicates the drug release mechanism.

\section{RESULTS AND DISCUSSION}

Three types of $\beta$-CD nanosponges prepared by varying the cross-linking density were used in this study. QU was loaded into three types of nanosponges by lyophilization method. The drug loading was

Table 1: Percent drug loading in nanosponges

\begin{tabular}{lll}
\hline S. No. & Name of the formulation & Drug loading (\%) \\
\hline 1 & QUNS1 & $16 \pm 0.61$ \\
2 & QUNS2 & $39 \pm 0.42$ \\
3 & QUNS3 & $29 \pm 0.47$ \\
\hline
\end{tabular}

All determinations were performed in triplicate and values were expressed as mean $\pm S D, n=3$. SD: Standard deviation, QUNS: Quercetin-loaded nanosponges

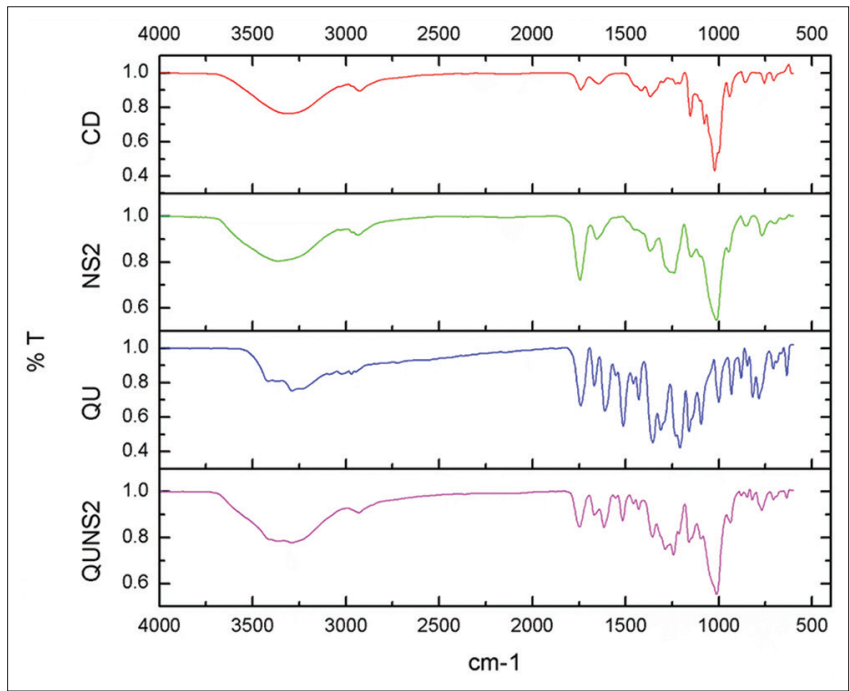

Fig. 1: Fourier transformed infrared spectra of $\beta$-cyclodextrin, plain nanosponges (NS2), quercetin (QU), and QUNS2 
determined for the estimation of drug association with nanosponges, i.e., the amount of drug encapsulated into nanosponges, calculated by analyzing a fixed amount of formulation. Percent drug loading into all the three types of nanosponges (QUNS1-QUNS3) is presented in Table 1.

Among all the three types of nanosponges (NS1-NS3), the loading efficiency was found to be higher in NS2 (1:4 $\beta$-CD: DPC) as much as $39 \% \mathrm{w} / \mathrm{w}$, while $16 \%$ and $29 \% \mathrm{w} / \mathrm{w}$ in NS1 and NS3 nanosponges, respectively. Due to the better solubilization and loading capacities, QUNS2 was selected for further studies.

\section{FTIR spectroscopy}

Fig. 1 shows a comparison of FTIR spectra of $\beta$-CD, NS2, QU, and QUNS2. NS2 showed a characteristic peak of carbonate bond at around $1720-1750 \mathrm{~cm}^{-1}$ which confirms the formation of CD-based nanosponges. In addition, the other characteristics peak of NS was found at $2918 \mathrm{~cm}^{-1}$ due to the $\mathrm{C}-\mathrm{H}$ stretching vibration, $1418 \mathrm{~cm}^{-1}$

Table 2: Particle size and zeta potential of quercetin complexes

\begin{tabular}{llll}
\hline Sample & $\begin{array}{l}\text { Mean } \\
\text { hydrodynamic } \\
\text { diameter } \pm \text { SD }(\mathbf{n m})\end{array}$ & $\begin{array}{l}\text { Polydispersity } \\
\text { index }\end{array}$ & $\begin{array}{l}\text { Zeta } \\
\text { potential }(\mathbf{m V})\end{array}$ \\
\hline NS2 & $408 \pm 18$ & $0.18 \pm 0.005$ & $-19.34 \pm 1.8$ \\
QUNS2 & $413 \pm 27$ & $0.25 \pm 0.005$ & $-20.55 \pm 1.4$ \\
\hline
\end{tabular}

All determinations were performed in triplicate and values were expressed as mean $\pm S D, n=3$. SD: Standard deviation, QUNS: Quercetin-loaded nanosponges
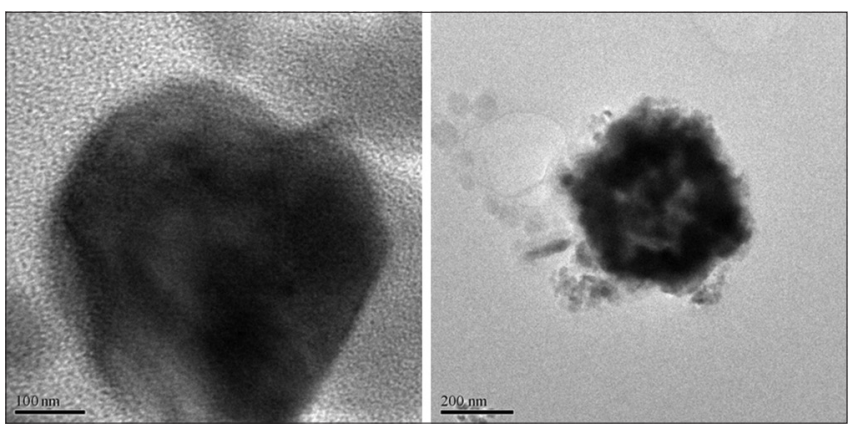

Fig. 2: Transmission electron microscopy images of plain nanosponge and quercetin-loaded nanosponges

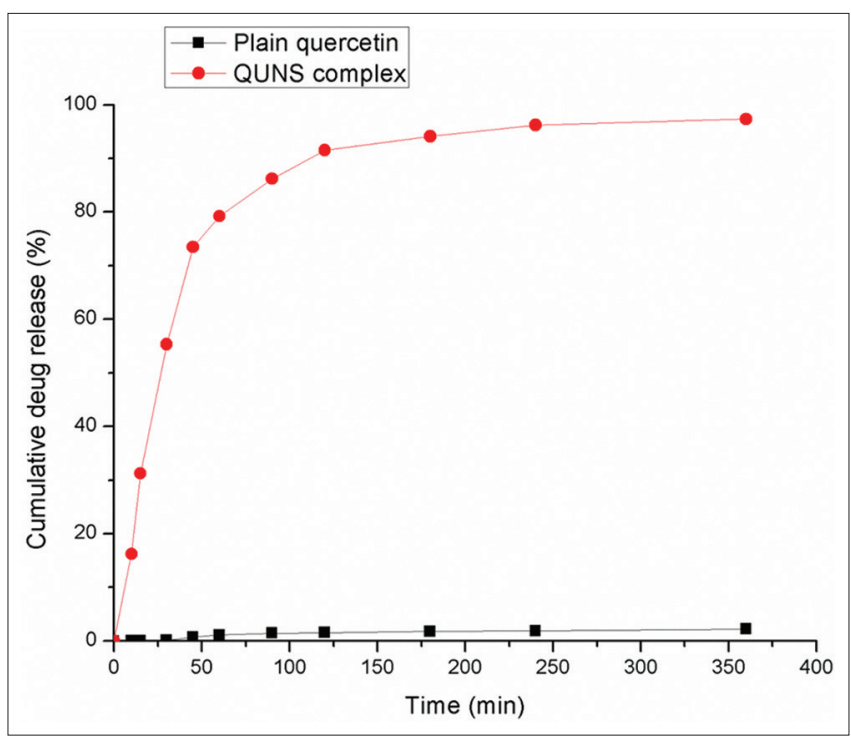

Fig. 3: Dissolution profile of original quercetin and quercetinloaded nanosponges due to $\mathrm{C}-\mathrm{H}$ bending vibration, and $1026 \mathrm{~cm}^{-1}$ due to $\mathrm{C}-0$ stretching vibration of primary alcohol. FTIR studies showed that there are weak interactions between NS and QU that were evident from broadenings and disappearance of the drug peaks in case of complexes. The comparison of FTIR spectra of QU and complex showed that there is a major change in the fingerprint region, i.e., $900-1400 \mathrm{~cm}^{-1}$. The main characteristic peaks of QU are at around 1663,1608, 1383, 1318, 1265, 1203, 1167, and $820-940 \mathrm{~cm}^{-1}$. These QU characteristic peaks were broadened or shifted in the formulations suggesting definite interactions between QU and NS. FTIR spectroscopy confirmed the formation of inclusion complex of QU with nanosponges.

Particle size, polydispersity index, and zeta potential determination The particle size analysis of NS2 and QUNS2 suspensions revealed that the average particle size measured by laser light scattering method is around 400-420 nm with low polydispersity index. The particle size distribution is unimodal and having a narrow range as seen in Table 2. A sufficiently high zeta potential indicates that the complexes would be stable and the tendency to agglomerate would be miniscule. A narrow PI means that the colloidal suspensions are homogenous in nature.

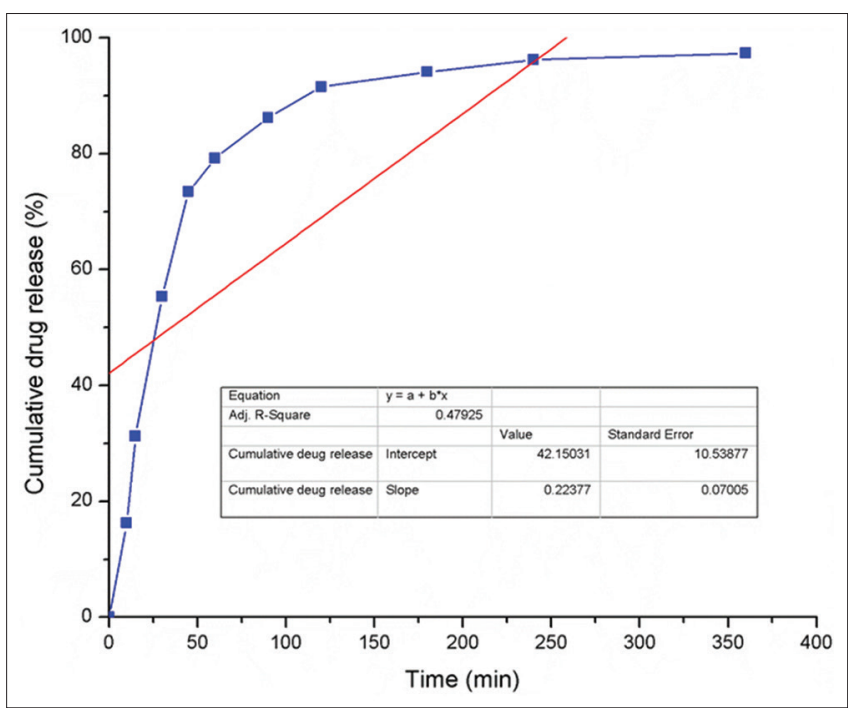

Fig. 4: Plot of zero-order release kinetics of the quercetin-loaded nanosponges formulation

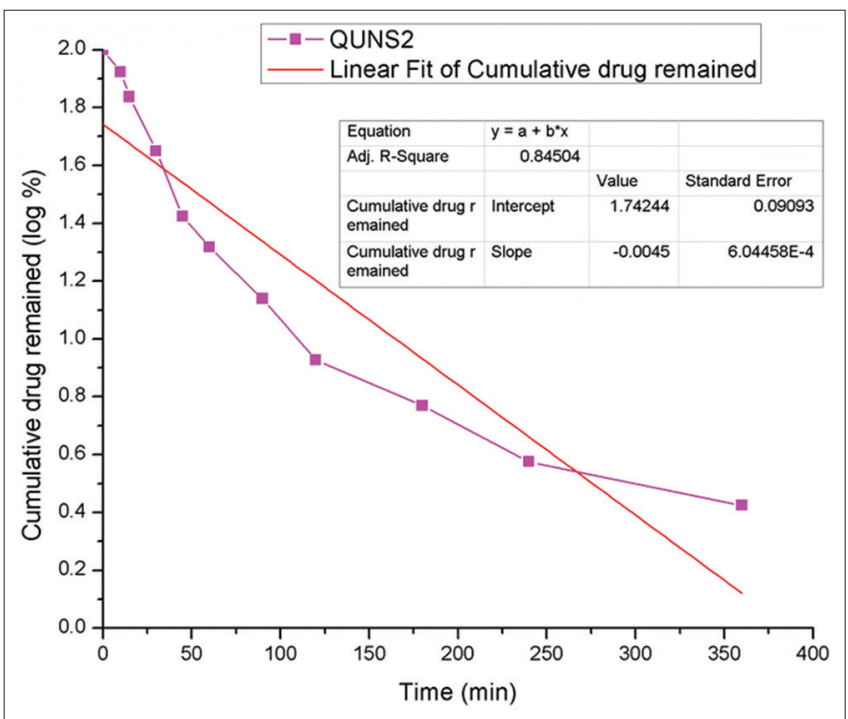

Fig. 5: Plot of first-order release kinetics of the quercetin-loaded nanosponges formulation 
Table 3: Release kinetics of optimized formulation of quercetin nanosponge complex

\begin{tabular}{|c|c|c|c|c|c|c|c|c|}
\hline \multirow[t]{2}{*}{ Formulation code } & \multicolumn{2}{|c|}{ Zero order } & \multicolumn{2}{|c|}{ First order } & \multicolumn{2}{|l|}{ Higuchi } & \multicolumn{2}{|c|}{ Korsmeyer-Peppas } \\
\hline & $\mathbf{R}^{2}$ & $\mathbf{n}$ & $\mathbf{R}^{2}$ & $\mathbf{n}$ & $\mathbf{R}^{2}$ & $\mathbf{n}$ & $\mathbf{R}^{2}$ & $\mathbf{n}$ \\
\hline QUNS & 0.47925 & 0.22377 & 0.84504 & 0.0045 & 0.76092 & 5.41628 & 0.976 & 0.9911 \\
\hline
\end{tabular}

QUNS: Quercetin-loaded nanosponges

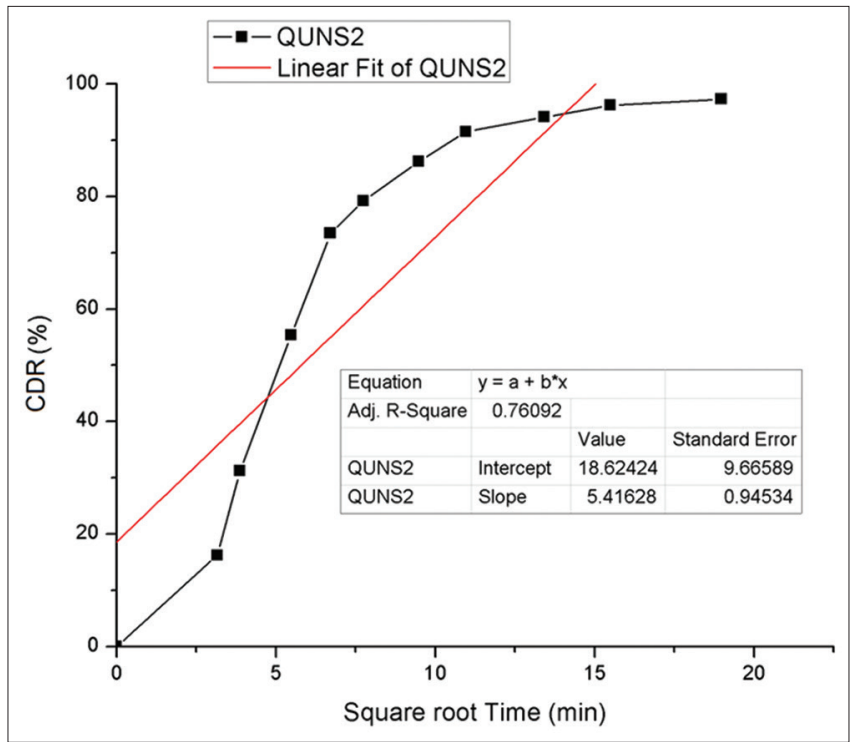

Fig. 6: Plot of Higuchi release kinetics of the quercetin-loaded nanosponges formulation

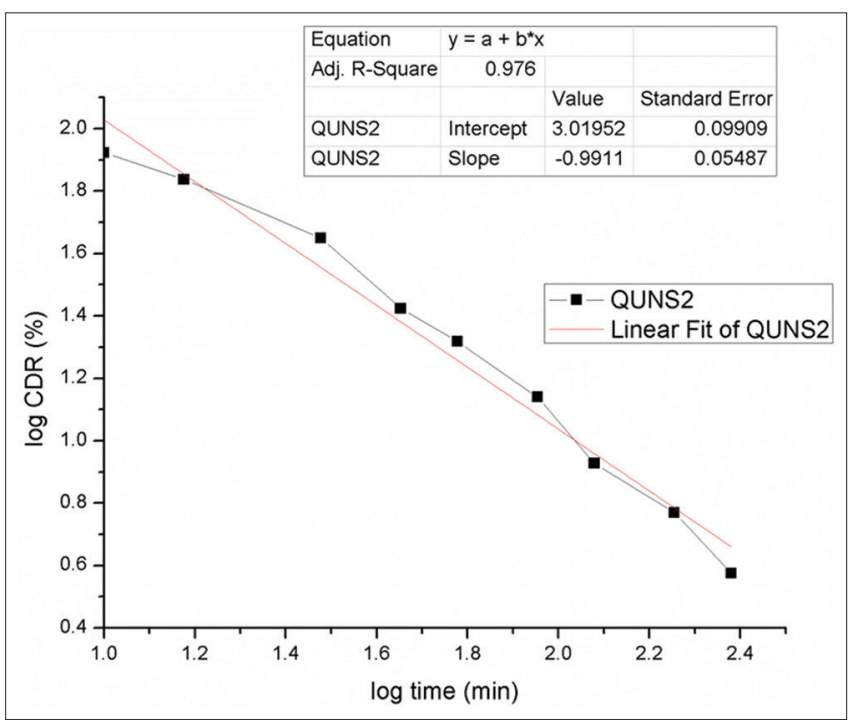

Fig 7: Plot of Korsmeyer-Peppas release kinetics of the quercetinloaded nanosponges formulation

\section{TEM}

TEM studies showed that the regular spherical shape and size of both the nanosponges that are unaffected even after drug encapsulation. TEM measurement revealed the porous structure of nanosponges as shown in Fig. 2. TEM studies revealed the porous structure of nanosponges and size of NS that are unaffected even after drug encapsulation (Table 3).

\section{In vitro release of $\mathrm{QU}$ from nanosponge formulations}

The dissolution profiles of plain QU and QUNS2 complex in simulated gastric medium are shown in Fig. 3. The in vitro release experiments showed a rapid and complete release of QU from nanosponges. From in vitro release, it was found that the complex showed a significant improvement in the rate of release as compared with the pure drug. The dissolution of pure QU is not even $2 \%$ in $120 \mathrm{~min}$, whereas the drug encapsulated in nanosponges showed faster release. An average 45-55\% QU was released within 30 min showing rapid burst release. This was normally attributed to the fraction of QU which was adsorbed or encapsulated as non-inclusion complex on the NS surface. On the addition of the formulation to the release medium, this fraction of QU was diffused rapidly into the surrounding liquid. The maximum release of QU after 120 min from QUNS2 was 91.54\%. After the initial effect, the release rate was found to be slower from the complex. The slower and sustained release of QU can be attributed to diffusion of the QU entrapped within the nanosponges as inclusion complex.

\section{Release kinetics}

Drug release data for the nanosponge formulation were fitted into various kinetic equations to find out the order and mechanism of drug release. The various release kinetic curves are shown in Fig. 4-7. The correlation coefficient showed that the release profile followed the Korsmeyer-Peppas model $\left(\mathrm{R}^{2}=0.976\right)$, the release exponent, $\mathrm{n}$ was found to be 0.9911 indicating non-Fickian (anomalous) transport. Kinetic analysis of drug release profiles showed that the systems predominantly released nimodipine in a non-Fickian (anomalous) with a strong correlation coefficient $\left(\mathrm{R}^{2}=0.94572\right)$ of Korsmeyer-Peppas model.

From the above results, it is apparent that the regression coefficient value closer to unity in case of Korsmeyer-Peppas model indicates that the drug release exponentially to the elapsed time. The data indicate a more linearity when plotted by the first-order equation. Hence, it can be concluded that the major mechanism of drug release follows first-order kinetics. Further, the translation of the data from the dissolution studies suggested possibility of understanding the mechanism of drug release by configuring the data into various mathematical modelings such as Higuchi and Korsmeyer-Peppas plots. Further, $\mathrm{n}$ value obtained from the Korsmeyer-Peppas plots, i.e., 0.9911 indicating non-Fickian (anomalous) transport; thus, it projected that delivered its active ingredient by coupled diffusion and erosion.

\section{CONCLUSION}

CD nanosponges have attracted the attention of many scientists due to their enhancing solubility of poorly water-soluble drugs. They can incorporate a wide range of hydrophilic and lipophilic drug molecules and they have the ability to form inclusion and non-inclusion complexes. The higher solubilization potential of nanosponges can be attributed to the formation of inclusion complex with drug as well as entrapment in the matrix form. From in vitro release, it was found that the complex showed a significant improvement in the rate of release as compared with the pure drug. Further, the translation of the data from the dissolution studies suggested possibility of understanding the mechanism of drug release by configuring the data into various mathematical modelings such as Higuchi and Korsmeyer-Peppas plots. Further, n value obtained from the Korsmeyer-Peppas plots, i.e., 0.9911 indicating non-Fickian (anomalous) transport; thus, it projected that delivered its active ingredient by coupled diffusion and erosion. ACKNOWLEDGMENTS

We would like to thank Prof. K. Venu Gopal Reddy (Director, CFRD, Osmania University) for assistance with FTIR analysis and Mr. Rajender (Centre for Nanoscience and Technology, JNTUH, HYD-85) for technical support with TEM. 


\section{AUTHORS' CONTRIBUTIONS}

All the authors have contributed equally.

\section{CONFLICTS OF INTEREST}

Declared none.

\section{REFERENCES}

1. Aluani D, Tzankova V, Yordanov Y, Zhelyazkova A, Georgieva E, Yoncheva K. Quercetin: An overview of biological effects and recent development of drug delivery systems. Pharmacia 2016;63:52-60.

2. Cirillo G, Vittorio O, Hampel S, Iemma F, Parchi P, Cecchini M, et al. Quercetin nanocomposite as novel anticancer therapeutic: Improved efficiency and reduced toxicity. Eur J Pharm Sci 2013;49:359-65.

3. Middleton E, Kandaswami C. The Impact of Plant Flavonoids on Mammalian Biology: Implications for Immunity, Inflammation and Cancer. London: Chapman and Hall; 1993.

4. Sánchez-Pérez Y, Carrasco-Legleu C, García-Cuellar C, PérezCarreón J, Hernández-García S, Salcido-Neyoy M, et al. Oxidative stress in carcinogenesis. Correlation between lipid peroxidation and induction of preneoplastic lesions in rat hepatocarcinogenesis. Cancer Lett $2005 ; 217: 25-32$

5. Chen J, Kang J, Da W, Ou Y. Combination with water-soluble antioxidants increases the anticancer activity of quercetin in human leukemia cells. Pharmazie 2004;59:859-63.

6. Anjaneyulu M, Chopra K. Quercetin attenuates thermal hyperalgesia and cold allodynia in STZ-induced diabetic rats. Ind J Exp Biol 2004:42:766-9.

7. Cemeli E, Schmid TE, Anderson D. Modulation by flavonoids of DNA damage induced by estrogen-like compounds. Environ Mol Mutagen 2004;44:420-6.

8. Reinboth M, Wolffram S, Abraham G, Ungemach FR, Cermak R. Oral bioavailability of quercetin from different quercetin glycosides in dogs. Br J Nutr 2010;104:198-203.

9. Graefe EU, Wittig J, Drewelow B, Riethling AK, Mueller S, Wukasch I, et al. Relative systemic availability of the flavonoids quercetin and rutin in humans. Arch Pharm Pharm Med Chem 1999;332:20.

10. Shaji J, Iyer S. Novel double loaded quercetin liposomes: Evidence of superior therapeutic potency against $\mathrm{CCl} 4$ induced hepatotoxicity a comparative study. Asian J Pharm Clin Res 2012;5:104-8.

11. Kumari A, Yadav SK, Pakade YB, Singh B, Yadav SC. Development of biodegradable nanoparticles for delivery of quercetin. Colloids Surf B Biointerfaces 2010;80:184-92.

12. Dhawan S, Kapil R, Singh B. Formulation development and systematic optimization of solid lipid nanoparticles of quercetin for improved brain delivery. J Pharm Pharmacol 2011;63:342-51

13. Li SJ, Liao YF, Du Q. Research and application of quercetin-loaded nano drug delivery system. Zhongguo Zhong Yao Za Zhi 2018;43:1978-84.

14. Lakshmi PK, Kumar MK, Aishwarya S, Shyamala B. Formulation and evaluation of ibuprofen topical gel: A novel approach for penetration enhancement. Int J Appl Pharm 2011;3:25-30.

15. Anjana MN, Sreeja CN, Joseph J. An updated review of cyclodextrins-an enabling technology for challenging pharmaceutical formulations. Int J Pharm Pharm Sci 2013;5:54-8.

16. Bipransh KT, Ravindra KZ, Kiran P, Ashis KN, Ranadhir C. Preparation and spectroscopic characterization of inclusion complex of 2-phenyl4h-benzo[d][1,3]oxazin-4-one and $\beta$-cyclodextrin. Int J Pharm Pharm Sci 2014;6:176-9.

17. Zheng Y, Chow AH. Production and characterization of a spray-dried hydroxypropyl-beta-cyclodextrin/quercetin complex. Drug Dev Ind Pharm 2009;35:727-34.

18. Carlotti ME, Sapino S, Ugazio E, Caron G. On the complexation of quercetin with methyl-b-cyclodextrin: Photostability and antioxidant studies. J Incl Phenom Macrocycl Chem 2011;70:81-90.

19. Challa R, Ahuja A, Ali J, Khar RK. Cyclodextrins in drug delivery: An updated review. AAPS PharmSciTech 2005;6:E329-57.

20. Szejtli J. Cyclodextrin in drug formulations Part I. Pharm Technol Int 1991;3:15-22.

21. Szente L, Szejtli J. Highly soluble cyclodextrin derivatives: Chemistry, properties, and trends in development. Adv Drug Deliv Rev 1999;36:17-28.

22. Lala R, Thorat A, Gargote C. Current trends in b-cyclodextrin based drug delivery systems. Int J Res Ayurveda Pharm 2011;2:1520-6.

23. Ansari KA, Vavia PR, Trotta F, Cavalli R. Cyclodextrin-based nanosponges for delivery of resveratrol: In vitro characterisation, stability, cytotoxicity and permeation study. AAPS PharmSciTech 2011;12:279-86

24. Swaminathan S, Pastero L, Serpe L, Trotta F, Vavia P, Aquilano D, et al. Cyclodextrin-based nanosponges encapsulating camptothecin: Physicochemical characterization, stability and cytotoxicity. Eur J Pharm Biopharm 2010;74:193-201.

25. Cavalli R, Trotta F, Tumiatti W. Cyclodextrin-based nanosponges for drug delivery. J Incl Phenom Macrocycl Chem 2006;56:209-13. 